\title{
A Review Literature on Mandibular Osteotomies
}

\section{Shashank Bhushan*}

Department of Oral and Maxillofacial Surgery, Buddha Institute of Dental Sciences and Hospital, Magadha University, India

*Corresponding Author: Shashank Bhushan, Department of Oral and Maxillofacial Surgery, Buddha Institute of Dental Sciences and Hospital, Magadha University, India.
Received: July 31, 2021

Published: September 14, 2021

(C) All rights are reserved by Shashank

Bhushan.

\begin{abstract}
The term "orthognathic" comes from the Greek ortho (straight) and gnathos (jaw). This straight jaw surgery developed only recently, largely Charrier J.-B. Orthognathic surgery of adults and facial aesthetics during the exuberant growth of medical and dental science over the last thirty years, when it emerged from maxillo-facial surgery, to become a sub-specialty of its own, orthognathic surgery was aided by collaboration of dento-facial orthopedic colleagues. This joint effort of surgeons and orthodontists has made it possible for team members to establish corrective treatment plans with precision and to execute them with rigor. Beauty, which is a central concept in all cultures, and physical appearance have always played key roles in the development of individual self esteem, in the establishment of interpersonal relations, and even the quality of life, are, accordingly carefully considered in these treatment plans. So the competence of orthognathic surgeon in no way limits itself to a mechanical occlusal or stomatological approach but integrates knowledge of the techniques of cosmetic surgery of the visage with procedures for correcting basal bone. The orthognathic surgeon is, above all, a surgeon of the face who is philosophically committed to incorporating surgery of the jaws with plastic surgery and reconstruction of the face.
\end{abstract}

Keywords: Mandibular Osteotomies; Orthognathic Surgery; Basal Bone; Plastic Surgery

\section{Introduction}

During the last three decades, remarkable advances have been made in surgical techniques and instrumentation for dentofacial surgery. Our knowledge and understanding of all aspects of orthognathic surgery have also grown over time. However, the basic surgical principles have more or less remained unchanged, despite technical innovations. Oral and maxillofacial surgery plays a central role in many aesthetic and functional procedures affecting the face and oral cavity. Since the first reported osteotomy performed on the mandible in the USA in 1849 a large number of improve- ments have been published in the fields of orthognathic surgery. A well-defined treatment plan is required to ensure a successful treatment outcome. The surgeon should develop and adopt a proper technique for each procedure; using the same surgical sequence enables assisting residents to anticipate each step, thus adopting skills and developing a routine. The leading surgeon should have explicit knowledge on each surgical step.

Born to a family of Irish farmers in western Pennsylvania on December 10, 1810, Simon P. Hullihen, MD, DDS, is considered by many to be the pioneer of orthognathic surgery [2]. In 1848 
he described the first mandibular osteotomy as part of a 3-stage operation for the treatment of a young woman severely burned as a child, who had contracture resulting in severe proganism and lower lip ectorpium. Hullihen's operation involved removing a Vshaped segment of the bone on either side of the pars alveolaris in the premolar region and a horizontal osteotomy in the body of the mandible, thus setting back the mandible in normal occlusion [2]. A similar technique, the anterior subapical osteotomy, is used today to address the protruding anterior dentoalveolar segment and to correct apertogenie, the open bite deformity of the mandible. For half a century not much interest in this operation was stirred, until around the turn of the century when Vilray Papin Blair of St Louis, Missouri, described an osteotomy of the body of the mandible in a patient with mandibular horizontal excess in whom a bilateral growth had occurred in the length of the horizontal ramus of the mandible, and on whom he operated on December 19, 1897 [3]. The objective of his technique was to shorten the horizontal ramus and to change the mental angle. He undertook the operation, with the patient under chloroform anesthesia, in which he introduced a double bladed saw that he had devised through an external approach through a submental incision [3]. Once the V-shaped osteotomies were performed and the anterior portion of the mandible rendered mobile, copper wires were used to pull the mobile segments together and decrease the "horizontal excess". Blair claimed that his procedure, if performed correctly, would address both horizontal excess and progeny [3]. The patient's "only abnormality" postoperatively, Blair stated, was total anesthesia of the lower lip [3]. In 1907, he proposed a horizontal osteotomy of the ramus using an extraoral approach to address both horizontal mandibular deficiency and excess.

It was not until 3 decades later that the intraoral approach came to be used for this osteotomy [3]. In 1906, von Eiselberg [4] proposed various surgical techniques for lengthening of the body of the mandible, but it was not until 1921 that the task was performed by Bruhn [5], who performed a vertical osteotomy of the body of the mandible followed by bone grafting. In 1912, Pickerill [6] described correction of progenie and apertogenie by staged wholethickness wedge resection of the body of the mandible 2 months apart in his book, Double Resection of the Mandible. Cohn-Stock [7], in 1921, proposed removing all the premolars followed by performing an oblique mandibular split circling the mental foramen to address uncomplicated apertogenie. Although he experimented on cadavers, he never performed the technique on a patient. In 1925, Limberg [8] developed the subcondylar osteotomy through the condylar neck using an extraoral approach "The farther we keep from the mouth cavity, the less dangerous is the operation. Therefore, the best method is to operate on the ascending ramus," he reasoned. His patient, "a girl of twenty," on whom he operated in December 1922 at the Medical Institute of Leningrad, experienced apertogenie secondary to underdeveloped bilateral rami leading to poor masticatory function and cosmesis. Limberg described his approach and osteotomy of the mandible through an incision 2 $\mathrm{cm}$ below the inferior edge of the mandibular body followed by an "oblique osteotomy of the ramus beginning from the incisura and slanting downward and backward to the lower part of the edge of the ramus," allowing for reposition of the mandible in the lower position. The neurovascular bundle was saved, and the wound was re-approximated using horsehair sutures. Limberg stated that an overcorrection may be necessary and the stylo mandibular ligament may need to be dissected during this approach. He later refined this technique and developed a longer cut that extended to the posterior border of the ramus, just above the angle. This technique was termed the posterior oblique vertical ramal osteotomy. In 1927, Wassmund [9] described the inverted L mandibular osteotomy. This involved vertical osteomies through the ramus and angle that then turned anteriorly below the coronoid process. Varaztad Hovhannes Kazanjian [10] MD, the prominent TurkishArmenian surgeon, developed the beveled horizontal osteotomy of the ramus in 1939 [11]. This extraoral approach cut the ramus at an angle to allow better preservation of the inferior alveolar neurovascular bundle. In 1942, Hofer [11] was first to describe the anterior horizontal osteotomy of the mandible. This was the first of many "sliding" osteotomies, and it was used by Gilles and Millard [12] for the treatment of a patient with Treacher-Collins-Franchetti syndrome in 1947. This procedure was then performed intraorally by Converse and Wood-Smith of New York in 1964 [13]. Dingman [14] described the mandibular step osteotomy of in 1944, and a decade later Caldwell and Letterman [15] described the intraoral vertical osteotomy of the ramus to address progenie. This setback technique was later further modified by Hebert., et al. [16] and Hibi and Ueda [17]. Caldwell., et al. [15] later described the "C osteotomy" of the mandible in 1968 to address the microgenic or retrogenic mandible. Schuchardt [18] of Itzehoe, Germany, a pupil of 
Wassmund at Rudolf Virchow Hospital, who later became a prominent maxillofacial surgeon in Berlin, introduced the intraoral approach for the "step" osteotomy of the vertical ramus in 1942. This osteotomy through the cortex with the medial cut above the lingula and the lateral cut $1 \mathrm{~cm}$ below it was followed by cortical splitting to allow for antero-posterior mobility of the distal segment. This was the first description of sagittal split osteotomy techniques for the mandible, more evolved versions of which comprise today's most commonly used mandibular osteotomy technique. In 1957, Trauner and Ob-wegeser [19] modified Schuchardt's sagittal split technique by carrying the lateral osteotomy lower to a point above the angle. This then allowed a wide-splitting osteotome to split the cortices and allow preservation of the inferior alveolar neurovascular bundle. This was followed by modifications by Dal Point (1961) [20], Hunsuck (1968) [21] and Epker and Fish (1977) [22]. In 1985, Dattilo., et al. [23] studied 20 patients who underwent an inverted L osteotomy for the treatment of open bite deformities. They found the procedure to have greater stability in the horizontal and vertical planes than in previously reported osteotomies. The first report of mandibular osteotomy for repair of trauma was done by Frankl [24] in 1968. He used an oblique osteotomy for the treatment of malunited fractures of the body bilaterally. In 1965 McCann., et al. [25] demonstrated the utility of the subcondylar osteotomy for repair of TMJ ankylosis. Celik., et al. [26] described a new genioplasty technique in 1999. In the splitting advancement genioplasty, a rectangular portion of the outer table of the mentum is split away, advanced, and then plated to the mandible. This technique is useful for advancement cases but not for reduction. It allows more contact surfaces for bony healing and a more natural projection of the mentum. In their article, Celik., et al. stipulate that this technique increases risk of infection owing to dead space from the advancement. In 2010, Schendel [27] proposed a new technique that minimizes aesthetic sequelae of the sliding osteotomies. His sagittal split genioplasty, which can be performed under local anesthesia, involves a sagittal split of the lateral one-third and two-thirds of the inferior chin. This then allows advancement of the inferior chin without a gap at the inferior border of the mandible. Accordingly, the hourglass deformity secondary to notching as seen in the sliding osteotomy is avoided.

Various mandibular osteotomes technique Bilateral sagittal split osteotomy

Bilateral sagittal split osteotomy (BSSO) is the most commonly performed jaw surgery, either with or without upper jaw surgery.
Indications for a bilateral sagittal split include horizontal mandibular excess, deficiency, and/or asymmetry [30]. The history of orthognathic surgery of the mandible started with Hullihen in 1846, who performed an osteotomy of the mandibular body for the correction of prognathism [30]. The earliest description of what would become the modern BSSO and the first intraoral approach to a ramal osteotomy was described in the German literature by Schuchardt in 1942 [18]. In 1954, Caldwell and Letterman described a vertical ramus osteotomy technique, which was shown to preserve the inferior alveolar neurovascular bundle [15]. The focus of innovation in mandibular surgery then migrated to Europe where Trauner and Obwegeser in 1957 described what would become today's BSSO [19]. The next several decades would see improvements and modifications to the procedure with the focus on decreasing relapse, improving healing, and decreasing complications. The main contributors to these improvements included Dal Pont (1961), Hunsuck (1968), and Epker (1977) [30].

\section{Complication}

Complications related to BSSO include bleeding from injury to the inferior alveolar artery or masseteric artery, unanticipated fractures and unfavorable splits, avascular necrosis, condylar resorption, malposition of the proximal segment and worsening of temporo-mandibular joint (TMJ) symptoms. The risk of injury to the inferior alveolar nerve is a significant consideration when performing a BSSO [30]. The incidence of transection is reported between 2 to $3.5 \%$ and the incidence of some form of long-term neurologic deficit is reported in 10 to $30 \%$ of patients, whether symptomatic or not [13]. When the sagittal split osteotomy is performed with an osseous genioplasty, nearly $70 \%$ of patients have some degree of neurosensory deficit at 1 year [14]. Fixation of the segments without proper seating of the condyles can result in condylar malposition, which can lead to rotation of the proximal segment and ultimate relapse, malocclusion, worsening of TMJ symptoms, and remodeling of the condylar head. Malocclusion in the form of an open bite is often the result of inadequate original fixation or hardware failure [30]. When noted intraoperatively, the fixation should be revised; when noted in the postoperative course films should be obtained to assess for hardware function. Small postoperative posterior open bites can often be managed orthodontically [30]. All orthognathic patients should be seen on a weekly basis following surgery if any signs of malocclusion develop and elastics adjusted appropriately to ensure healing in the proper occlusion. Proximal

Citation: Shashank Bhushan. "A Review Literature on Mandibular Osteotomies". Acta Scientific Dental Sciences 5.10 (2021): $29-39$. 
segment fractures occur most often as a result of failure to completely cut the inferior border; this results in a fracture line that propagates along the buccal side of the inferior border [30]. As the two fragments are split and this is noted, the inferior border should be recut. Impacted third molars are another cause of unfavorable fractures and should ideally be removed 6 months to 1 year prior to mandibular surgery [30]. When an impacted third molar must be removed at the time of surgery, care should be taken to not use excessive force. Cutting the tooth into smaller fragments will facilitate this. Since the modern era of screw fixation, the incidence of lingual nerve injury has declined and become an uncommon complication following a BSSO [30]. However, several cases have been reported in the literature. In most instances, lingual nerve paresthesia spontaneously resolves, but Pepersack and Chausse reported a 3\% neurosensory deficit at 5 years [42]. Most cases were due to wire or bicortical screw placement near the superior border of the mandible in the region of the third molar.

Temporomandibular dysfunction (TMD) is a common finding in the general population, with a reported incidence between $20 \%$ and 25\% [30]. The incidence of preoperative TMD in the orthognathic population is reported to be between 16 and 50\% [30]. The most frequent symptoms identified were pain and clicking of the TMJ. Although the literature has a wide variation in the rates of symptom improvement, most studies have shown that the majority of patients has improvement in their symptoms with only a small percentage experiencing worsening of symptoms [30]. Decreased mobility after a BSSO is not an uncommon postoperative problem. It is most frequently attributable to prolonged immobility that results in fibrosis and atrophy of the muscle and connective tissue of the masticatory system [30]. The incidence of hypomobility after a BSSO has declined with the use of rigid fixation, as prolonged periods of maxilla-mandibular fixation are not necessary [30]. With the institution of a program of active rehabilitation, most patients return to preoperative inter-incisal opening within 3 months [30]. Intraoperative serious hemorrhage is a rare complication during a BSSO [30]. Maintaining the surgical dissection subperiosteally and adequate retraction of soft tissue prevent minor intraoperative oozing and most cases of major hemorrhage [30]. Minor hemorrhage from tearing of the periosteum can be controlled with electrocautery, pressure or additional vasoconstrictive agents [30].

\section{Intraoral vertical mandibular ramus osteotomy}

It is traditionally used when large setback of the mandible is required as well as for the correction of the severe mandibular asymmetry [34].

This procedure has several advantages over BSSO:

1. It has less inferior nerve morbidity.

2. It can performed at an out patient basis.

3. It cause less flaring of the proximal segment.

4. There is no need for rigid fixation.

5. Existing tmj dysfunction can be treated concurrently.

Disadvantages:

1) The need for postoperatively maxillomandibular fixation.

2) The need for a strict protocol of active physiotherapy for at least 2 week after release of fixation.

\section{Extraoral}

In the early 1950s, Caldwell and Letterman [15] popularized an osteotomy performed in the ramus of the mandible for the correction of mandibular excess. This technique is mainly used for the correction of asymmetry of the mandible [31]. In this procedure, the lateral aspect of the ramus is exposed through a submandibular incision. The incision is marked about $2 \mathrm{~cm}$ below the inferior border of the mandible base, in the angle region. The length of the skin incision is about $4 \mathrm{~cm}$ long [31]. Dissection is made through the skin and all the covering soft tissues over the platysma are carefully undermined. An incision is then performed on the platysma to the next layer as deep as to the superficial layer of the deep cervical fascia. The marginal mandibular branch of the facial nerve passes through this layer. Thus, extreme care should be taken [31]. As soon as this layer is dissected, the masseter muscle will be exposed which can be cut with a knife. From here, dissection can be continued posteriorly to the gonial notch and superiorly subperiosteally to expose the ascending ramus laterally. Before starting the osteotomy, the medial pterygoid muscle should be released from the medial part of the angle [31].

The ramus is osteotomized in a vertical direction, posterior to the foramen where the mandibular inferior nerve enters [31]. The 
cut can be done superiorly from the sigmoid notch to the angle of the mandible. This osteotomy is suitable for posterior repositioning of the mandible It is also possible to perform an inverted L osteotomy, where a horizontal cut from the anterior section of the ascending ramus is made just below the coronoid process extending to the vertical osteotomy from the angle of the mandible [31]. The entire body and anterior ramus section of the mandible are moved posteriorly, which places the teeth in proper occlusion [31]. The proximal segment of the ramus that is attached to the condyle will overlap the anterior part of the jaw that includes the teeth and will be stabilized during the healing phase with wiring or plating of the bone segments combined with using elastic mandibulomaxillary fixation [31].

\section{Intraoral}

A similar technique can be done through an intraoral incision and an angulated oscillating saw or piezo saw [31]. The design of the osteotomy is identical to that performed through an extraoral incision. The incision is performed via an intraoral entrance. This procedure has, at least, two advantages namely elimination of the risk of damage to the facial nerve and elimination of the risk of a visible scar postoperatively [31]. The main disadvantage with the intraoral approach is that it is difficult to perform since visibility is limited [31]. Additionally, fixation of the fragments is difficult. Previously, it was common to use mandibulomaxillary fixation (MMF) for 5 - 7 weeks postoperatively when this approach was used. A rigid, internal fixation can be used but MMF must be considered for a short period [31].

\section{Genioplasty}

Genioplasty is used to address numerous facial concerns from a facial balancing procedure in adjunction with orthognathic surgery to assisting with soft tissue contours and chin-neck enhancement for patients undergoing elective facial surgery [49]. Over the years many authors have proposed different studies leading to various advances in the method of genioplasty in order to treat numerous types of chin augmentation. In 1934, Aufricht described the use of nasal cartilage as a means for chin augmentation. Trauner and Obwegeser in 1957, published the first article on intraoral sliding osseous genioplasty, which is still used throughout the world today [19]. In 1942, Hofer first described advancement genioplasty through an external approach [11], later in the late 1950s Trauner and Obwegeser described an intraoral approach, Converse and
Wood-Smith. In the 1960s Converse and Wood-Smith, as well as Hinds and Kent, described the versatility of the sliding genioplasty. In the 1970s Gonzales-Ulloa, Loeb and Field described various methods to address-witch's chin and deep submental folds [49]. 1980s the introduction of the use of hydroxyapatite as an inlay or onlay graft to augment the lower facial height in the osteotomized chin [25]. In the late 1990s to 2007, Zide and his colleagues wrote a series of articles stating numerous contemporary aspects of genioplasty approaches, evaluation, complications, and refinements that serve as a strong foundation for surgeons undertaking this procedure [49].

\section{Horizontal advancement}

Anteroposterior chin deficiency (microgenia)- Anteroposterior augmentation of the chin by sliding genioplasty is the most common operation to correct an anteroposterior deficient chin [49]. The sliding genioplasty for chin augmentation has pronounced advantages above the use of alloplastic materials. The inferior segment is brought forwards, and wires are used to fix the lingual cortex to the buccal cortex of the main body of the mandible. Or it can also be done by placing bent mini plates [49].

\section{Horizontal set-back}

Anteroposterior excess (macrogenia) is mainly caused due to the skeletal prominence of the symphysis, however, excessive soft tissue thickness can also lead to an unesthetic chin projection [49]. When the chin is reduced anteroposteriorly, the clinician should take care not to reduce or flatten the labiomental fold resulting in unesthetic chin shape [49]. The proximal tips of the mobilised fragment are reduced in order to ensure a smooth transition along the inferior border [49].

\section{The tenon technique}

Michelet., et al. described the tenon technique of genioplasty $U$ shaped monocortical osteotomy cut is created centrally in the symphysis [49]. Below the mental nerves lateral extensions are developed, which connect to the superior limbs of the U Full thickness osteotomies are completed on the lateral extensions only through the lingual cortex on the superior aspect of the $U$ The resultant full thickness of bone behind the tenon allows for a better positioning and lag screw fixation. In case posterior movement is desired, the $\mathrm{U}$ is inverted and the tenon is in the inferior fragment [49]. 


\section{Vertical reduction}

Vertical chin excess: When the lower facial height has increased we should differentiate between vertical mandibular excess and vertical maxillary excess. In cases where the maxilla is vertically excessive: the interlabial gap has to be increased; the upper incisor exposure under the upper lip is increased and patient often has a gummy smile [49]. The height of the chin will be excessive when: the lower two thirds (Stm-Me) of the lower facial half (Sn-Me) is more than the normal ratio of 1:2 = Sn-Stm: Stm-Me, while the lower half of the facial ( $\mathrm{Sn}-\mathrm{Me}$ ) will be excessive corresponding to the upper half $(\mathrm{N}-\mathrm{Sn}<\mathrm{Sn}-\mathrm{M}$ e) [49].

The vertical height changes are effected by altering the angle of the osteotomy [49]. The magnitude of vertical dimensional change is proportional to the direction and amount of the horizontal movement [49]. If we want to shorten the chin without horizontal changes, a wedge reduction is usually indicated, which can be performed using horizontal osteotomy or tenon technique. It is easier to do the wedge ostectomy from the superior stable fragment [49].

\section{Vertical augmentation}

Vertical chin deficiency: Decrease in the lower facial height relative to the midface height is caused due to a vertical deficiency of the anterior mandible [49]. Vertical chin deficiency should be differentiated from deep bite cases and vertical maxillary deficiency [49]. In all three dentofacial deformities will be clinically and cephalometrically as a diminutive or "squashed" lower facial third, vertically shorter than the middle third. The lower two thirds (Stm - Me) of the lower facial height ( $\mathrm{Sn}-\mathrm{Me}$ ) is shorter in relation to the upper third (Sn - Stm) [49]. This is indicated when the deficit is in the mandibular alveolus or the symphysis, the lower facial height is to be increased [49]. Vertical augmentation is achieved by alloplastic implant placement between the osteotomised segments or by interpositional grafting or altering the angle of the horizontal cut [49].

\section{Transverse chin deformities}

Transverse deficiency: The chin can be widened in the posterior area by cutting the chin segment in the midline and rotating the segments outward, while the anterior part of the chin can be widened by lateral repositioning of the segments and also by placement of a bone graft in the defect in the midline [49].
Transverse excess: The chin may be made narrow or made more tapered by sectioning the chin segment in the midline and removing a triangular section of the bone from the lingual aspect [49]. Placing a bone plate on the anterior surface of the segment before mobilization will allow the segment to be "bent" narrower [49]. A very broad, square, chin can be narrowed by removing a rectangular section of bone in the middle of the chin segment. The segments are finally moved medially and fixated [49].

\section{Segmentary subapical mandibular osteotomy}

Segmentary subapical osteotomy is a technique for orthognathic surgery used in cases of dentoskeletal malocclusions that cannot be dealt with only by conventional orthodontic treatment. Its use has been focused to achieve occlusal stability through dentoalveolar movement. Using this technique a favorable occlusal relationship may be established thus allowing good interaction between the dental arches at mastication during mandibular movements. These dental-alveolar changes are performed in an axial, anteroposterior, transverse and vertical direction with an alveolar-dental impact. Intrusion movements are useful when you need to mobilize more than $2 \mathrm{~mm}$ in adult patients. Hofer in 1942 and Kole in 1959 popularized the subapical technique for segments of both maxillaries in the anterior and posterior sectors. MacIntoch described the total mandibular alveolar osteotomy in 1974 for the correction of anterior open bite. Eliades and Hegdvedt reported in 1996 the first combination of sagittal osteotomy with complete subapical osteotomy for the successful correction of class II malocclusions. The biological bases of maxillary osteotomy were described by Obwegeser, Kole and Bell in their different articles. Kulbersh and Pangrazio compared total subapical osteotomy with bilateral sagittal osteotomy for the correction of class II dentofacial deformities showing for both techniques long-term stability.

\section{Lateral body osteotomy of the mandible}

This osteotomy is widely used in selected cases of mandibular prognathism. This method is used especially when first or second premolars are missing or are planned to be extracted, and by using this method, a prosthetic reparation of intact teeth can be avoided. It is also desirable to use this method in the case of an anterior open bite, excess growth of the mandible with negative overjet located in the anterior dentoalveolar area of the mandible, to treat mandibular dental arch asymmetry. This method can be used to 
reposition the anterior section of the mandible in every desirable direction, such as for posterior and superior repositioning. The method can be used for a block osteotomy or segmental or total alveolar osteotomy

\section{Anterior subapical osteotomy}

Anterior subapical osteotomy is suggested when the skeletal class I relation exists, and deformities in the front part of the mandible cannot be treated by orthodontic treatment. Some of the indications are as follows [30]:

- Excess growth of the mandible with negative overjet located in the anterior dentoalveolar process of the mandible,

- The negative curve of Spee,

- Some particular types of open bite,

- Mandibular dental arch asymmetry,

- When a lateral body osteotomy is planned, but the position of the chin is satisfactory.

Anterior subapical osteotomy method can be used to reposition the anterior section of the mandible in every desirable direction, such as posterior and superior repositioning [30]. Modification by KOLE Bone gaps caused by movement of the segment, especially by vertical movement necessary for the closure of an anterior open bite, should be grafted. The use of cortical bone from the symphysis, as advocated by Kole.

\section{Posterior subapical osteotomy}

This method can be used for the correction of super eruption of mandibular posterior teeth or ankylosis of the posterior teeth [30]. A transoral incision is performed at the anterior border of the vertical ramus and is carried forward to the canine area [30]. The incision is made around the margins of the teeth with starts one tooth behind the proposed osteotomy anteriorly and posteriorly [30]. Two vertical, oblique incisions are connected to the horizontal. The periosteal attachment is released, but the inferior border is untouched to keep the soft tissue contour unchanged, and also to keep the blood circulation undisturbed [30]. Again, the neurovascular bundle is identified and the incision is extended carefully in an anterior direction to the premolar area [30]. The osteotomy is horizontally placed subapical, about $5 \mathrm{~mm}$ from the root apices, and special care must be taken to preserve the mandibular canal [30]. The horizontal osteotomy is connected with two vertical oste- otomies between the first molar and second premolars [30]. Interdental cuts should as always be done carefully with a chisel to avoid injury to the roots. The posterior segment can then be repositioned superiorly [30].

\section{Inverted L osteotomy}

The inverted L osteotomy is frequently applied to class II skeletal deformities that possess a short vertical ramus height and con-cominant high mandibular plane angle [32]. The skeletal correction often requires significant counter clockwise movement to improve the projection of the lower facial third. This osteotomy and subsequent fixation is typically performed through a submandibular (Risdon) skin incision [32].

\section{Indications for inverted L osteotomy}

The sagittal ramus osteotomy is the most common orthognathic surgical technique performed on the mandible as it can be applied to a wide array of skeletal deformities (hypoplasia, hyperplasia and asymmetry) and subsequently advanced, setback or repositioned to establish symmetry [32]. Mandibular advancement for deficiency of the lower jaw is classically addressed via a sagittal ramus osteotomy as bone continuity is maintained and fixation over the osteotomies generally routine through either bicortical screw or plate fixation. Surgical indications for the inverted L osteotomy include large counterclockwise rotations of the maxillomandibular complex to create projection of the lower facial third for the establishment of improved facial balance and proportion when alteration of ramus anatomy is present [12]. When performing any mandibular osteotomy, including the inverted L the anatomy and stability of the mandibular condyle should be taken into account [32]. Those patients with degenerative joint disease (DJD) or juvenile rheumatoid arthritis (JRA) that have markedly distorted condylar anatomy, but have preservation of joint functionality are candidates for correction of the dentofacial deformity through an osteotomy as opposed to joint replacement [32].

\section{Advantages}

The advantages of performing the inverted L osteotomy intraorally are numerous.

Avoid a large neck skin incision for access. There is a streamlined flow using an isolated transoral approach that lends itself to improved intraoperative efficiency [32]. Stepwise completion of 
the osteotomy, IMF and subsequent rigid fixation is similar to that of more traditional orthognathic surgical approaches. Virtual surgically planned cutting guides can assist with osteotomy guidance, helpful in the hands of the novice or surgeon in training [32].

The quantification of the osteotomy gap preoperatively and fabrication of bone grafting template helps the mitering of an allograft or autograft [32].

Patient specific custom mandibular reconstruction plates allow for unique designs, shapes and contours often unable to be achieved through traditional bends of a "stock" reconstruction plate [32]. The custom nature allows the avoidance of screw interference with tooth roots, nerves, osteotomies, and implants [32]. The plate can be designed to customize the strength through control of thickness and width between holes or spans over the osteotomy [32].

\section{Disadvantages}

Although the advantages of using VSP and CMRP outweigh the disadvantages, there are several areas that require discussion [32]. The additional time requirement needed for virtual planning the correction of the dentofacial deformity and the associated planning of the pre-bent custom reconstruction plate is more than for standard VSP orthognathic case [32]. There is an increased cost incurred beyond the standard VSP orthognathic preparation limited to intermediate and final splints [32]. Surgeons would argue that the virtual planning through cutting guides and occlusal splints for efficiency and accuracy is as valuable as the fixation placed on typical orthognathic cases [32]. Even with the advent of cutting jigs, bone graft templates and pre-bent custom plates the surgical technique remains a technically complex procedure [32]. The limited visibility, confined access and unconventional orthognathic techniques can be challenging in the hands of surgeons of all experience levels [32]. Focusing on mandibular surgery first may concerns surgeons who are accustom to addressing the maxillary position initially [32]. As with conventional orthognathic surgery, the inability to satisfactorily reposition the mandible when staged first results in the necessity to abandon moving on the maxilla and finalizing the case as planned [32].

\section{Chin-Wing osteotomy}

In 2009, Albino Triaca described a different osteotomy, called chin wing osteotomy, for the correction of aesthetic dislocation of the lower face, either in the anterior part of the mandible [31]. The name of the approach was coined due to the fact that the shape of the mandible base is like a wing [31].

Mandibular osteotomy for expanded transoral robotic surgery (Motors)

Transoral Robotic Surgery (TORS) has revolutionized the treatment of head and neck cancer, specifically for malignant lesions of the oropharyngeal region [33]. Recent studies have shown that this approach is a safe procedure and can provide favorable clinical and functional outcomes with respect to traditional approaches [33]. These investigations have noted several advantages relative to traditional transmandibular approaches which include faster recovery of swallowing and vocal function, decreased need for reconstruction, decreased incidence in aspiration pneumonia and decreased length of hospitalization [1,2]. Current TORS applications in the head and neck include lesions of the laryngopharyngeal subsites including the base of tongue, tonsillar fossa, palate, posterior pharynx and epiglottis. Recent literature has demonstrated that salvage surgery with TORS for recurrent oropharynx tumors versus open surgery has superior outcomes with respect to function, morbidity and operative time [33]. Despite transoral surgery being the favored salvage option, some patients are unable to undergo transoral procedures due to limited access [33]. In these patients, the ability to gain appropriate exposure to the lesion often dictates which patients can undergo TORS/TLMS (transoral laser microsurgery), rather than oncologic considerations alone [33]. Suboptimal exposure leads to increased operative times, greater risk of surgical complications, and the possibility of inadequate surgical resection margins [33]. In addition, due to similar anatomic constraints our technique can applied to any transoral surgery requiring increased access such as in the case of TORS or TLMS [33]. This investigation is designed to test the hypothesis that mandibular osteotomies can expand access to the oral cavity, oropharynx, hypopharynx, and supraglottis [33].

\section{Conclusion}

Dentofacial deformities affect approx. $20 \%$ of the population. Patients with dentofacial deformities may demonstrate various degrees of functional and esthetic compromise. Such malformation may be isolated to one jaw or they may extend to multiple craniofacial structures. They may occur unilaterally or bilaterally and may be expressed to varying degrees in the vertical, horizontal and transverse facial planes. During the last three decades, remarkable 
advances have been made in surgical techniques and instrumentation for dentofacial surgery. However, the basic surgical principles have more or less remained unchanged, despite technical innovations. Oral and maxillofacial surgery plays a central role in many aesthetic and functional procedures affecting the face and oral cavity. A well-defined treatment plan is required to ensure a successful treatment outcome. The surgeon should develop and adopt a proper technique for each procedure; using the same surgical sequence enables assisting residents to anticipate each step, thus adopting skills and developing a routine. The leading surgeon should have explicit knowledge on each surgical step. Osteotomies of jaw must be performed in a safe way and preferably intraorally to avoid scars on the face and at the same time provide adequate exposure to the skeleton. Essential structures must be preserved, and provision for appropriate nutrition postoperatively should be considered.

\section{Bibliography}

1. Aziz SR and Simon P. "Hullihen and the origin of orthognathic surgery". Journal of Oral and Maxillofacial Surgery 62.10 (2004): 1303-1307.

2. Hullihan SP. "Case of elongation of the under jaw and distortion of the face and neck, caused by a burn, successfully treated". American Journal of Dental Science 9 (1849): 157-165.

3. Blair VP. "Operations on the jaw bone and face”. Surgery, Gynecology and Obstetrics 4 (1907): 67-78.

4. Von Eiselberg A. "Uber plastic bei ektropium des unterkeifers (progenie)". Wiener klinische Wochenschrift 19 (1906): 15051508.

5. Bruhn C. "Management of Gunshot Injuries of The Jaw, Based on the Experience of the Jaw Hospital in Du"sseldorf [in German]". Wiesbaden, Germany: Verlag JF von Bergmann 19151917.

6. Pickerill HP. "Double Resection of the Mandible”. Philadelphia, PA: SS White Dental Manufacturing Co (1912).

7. Cohn-Stock G. "Die chirurgische Immediatregulierung der Kiefer, speziell die chirurgische Behandlung der Prognathie". Berlin, Germany: Vjschr Zahnheilk 37 (1921): 320.
8. Limberg A. "Treatment of open-bite by means of plastic oblique osteotomy of theascending rami of the mandible". Dent Cosmos 67 (1925): 1191-1200.

9. Wassmund M. "Frakturenund Luxationen des Gesichtsscha"dels unter Beru"cks DKomplikationen d". Hirnscha"dels Meusser. Berlin, Germany: Meusser (1927).

10. Kazanjian VH. "The surgical treatment of prognathism; an analysis of sixty-five cases". The American Journal of Surgery 87.5 (1954): 691-700.

11. Hofer D. "Operation der Prognathie und Mikogenie". Dtsch Zahn Mund Kieferheilkd 9 (1942): 121.

12. Gilles HD and Millard DR Jr. "The Principles and Art of Plastic Surgery". Boston, MA: Little Brown and Co Inc; (1957): 4.

13. Converse JM and Wood-Smith D. "Horizontal osteotomy of the mandible". Plastic and Reconstructive Surgery 34 (1964): 464471.

14. Dingman RO. "Osteotomy for correction of mandibular malrelation of developmental origin". Journal of Oral and Maxillofacial Surgery 2 (1944): 239-247.

15. Caldwell JB and Letterman GS. "Vertical osteotomy in the mandibular raml for correction of prognathism". Journal of Oral and Maxillofacial Surgery 12.3 (1954): 185-202.

16. Hebert JM., et al. "Correction of prognathism by an intraoral vertical subcondylar osteotomy". Journal of Oral and Maxillofacial Surgery 28.9 (1970): 651-653.

17. Hibi $\mathrm{H}$ and Ueda $\mathrm{M}$. "A technique for ensuring accurate bone cuts in the intraoral verticalramus osteotomy". Journal of Oral and Maxillofacial Surgery 53.12 (1995): 1480-1481.

18. Schuchardt K. "Ein Vorschlag zur Verbesse- rung der Drahtschienenverbiinde”. DtschZahn Mund Kieferheilk 24 (1956): 3944.

19. Trauner R and Obwegeser H. "The surgical correction of mandibular prognathism and retrognathia with consideration of genioplasty, I: surgical procedures to correct mandibular prognathism and reshaping of the chin". Oral Surgery, Oral 
Medicine, Oral Pathology and Oral Radiology 10.7 (1957): 677689.

20. Dal Pont G. "Retromolar osteotomy for the correction of prognathism". Journal of Oral Surgery, Anesthesia, and Hospital Dental Service 19 (1961): 42-47.

21. Hunsuck EE. "A modified intraoral sagittal splitting technic for correction of mandibular prognathism". Journal of Oral and Maxillofacial Surgery 26.4 (1968): 250-253.

22. Epker BN and Fish L. "Surgical-orthodontic correction of open-bite deformity". American Journal of Orthodontics 71.3 (1977): 278-299.

23. Dattilo DJ., et al. "The inverted L osteotomy for treatment of skeletal open-bite deformities". Journal of Oral and Maxillofacial Surgery 43.6 (1985): 440-443.

24. Frankl Z. "The place of oblique osteotomy in the treatment of malunited fractures of the body of the mandible". British Journal of Oral Surgery 5.3 (1968): 245-250.

25. McCann CF., et al. "Bilateral subcondylar osteotomy: report of case”. Journal of Oral and Maxillofacial Surgery 23 (1965): 240244.

26. Celik M., et al. "Splitting advancement genioplasty: a new genioplasty technique". Annals of Plastic Surgery 43.2 (1999): 148-153.

27. Schendel SA. "Sagittal split genioplasty: a new technique". Journal of Oral and Maxillofacial Surgery 68.4 (2010): 931-934.

28. Bell WH. "Modern practice in orthognathic and reconstructive surgery". Philadelphia: WB Saunders Company (1992).

29. Thierry M and Charrier JB. "Les corticotomies alve' olaires, principes et applications cliniques". International Orthodontics 6 (2008): 343-354.

30. Laura A Monson., et al. Department of Surgery, Baylor College of Medicine, Houston, Texas Semin Plast Surgery 27 (2013): 145-148.

31. Kashani $\mathrm{H}$ and Rasmusson L. "Osteotomies in Orthognathic Surgery". A Textbook of Advanced Oral and Maxillofacial Surgery 3 (2016).
32. Peter B Franco. "Brian B Farrell Inverted L osteotomy: a new approach via intraoral access through the advances of virtual surgical planning and custom fixation 2.1 (2016): 1-9.

33. Alfred Marc C Iloreta., et al. "Mandibular Osteotomy for Expanded Transoral Robotic Surgery: A Novel Technique". Laryngoscope 124 (2014): 1836-1842.

34. Reyneke JP. “The bilateral sagittal split mandibular ramus osteotomy - surgical manual, $2^{\text {nd }}$ edition". Jacksonville, FL: Biomet Micro Fixation (2008).

35. Hall HD., et al. "Evaluation and refinement of the intra-oral vertical subcondylar osteotomy". Journal of Oral and Maxillofacial Surgery 33 (1975): 333.

36. Precious D. “Genioplasty”. In: Fonseca RJ, Marciani RD, Turvey TA, (Edition.), Oral and maxillofacial surgery, vol. III, 2nd edition. St Louis, MO: Elsevier (2009): 137-154.

37. Schmoker R., et al. "[Functionally stable osteosynthesis of the mandible by means of an excentric-dynamic compression plate. Results of a follow-up of 25". SSO Schweiz Monatsschr Zahnheilkd 86.2 (1976): 165-185.

38. Manikandhan Ramanathan., et al. "A Modified Kole's Osteotomy for Correction of Anterior Open Bite and Macrogenia in a Cleft Patient". Craniomaxillofacial Trauma Reconstruction 6 (2013): 57-60.

39. Takahashi H., et al. "Three lateral osteotomy designs for bilateral sagittal split osteotomy: biomechanical evaluation with three-dimensional finite element analysis". Head and Face Medicine 6 (2010): 4.

40. D'Agostino A., et al. "Complications of orthognathic surgery: the inferior alveolar nerve". Journal of Craniofacial Surgery 21.4 (2010): 1189-1195.

41. Van Sickels JE., et al. "Effects of age, amount of advancement, and genioplasty on neurosensory disturbance after a bilateral sagittal split osteotomy". Journal of Oral and Maxillofacial Surgery 60.9 (2002): 1012-1017.

42. Pepersack WJ and Chausse JM. "Long term follow-up of the sagittal splitting technique for correction of mandibular prog- 
nathism". Journal of Oral and Maxillofacial Surgery 6.2 (1978):

117-140.

43. Thilander B., et al. "Prevalence of temporomandibular dysfunction and its association with malocclusion in children and adolescents: an epidemiologic study related to specified stages of dental development". The Angle Orthodontist 72.2 (2002): 146-154.

44. Bell WH and Dann JJ. "Correction of dentofacial deformities by surgery in the anterior part of the jaws". American Journal of Orthodontics and Dentofacial Orthopedics 64 (1973): 1621187.

45. Brusati R and Gianni AB. "Anterior mandibular apical base augmentation in the surgical orthodontic treatment of mandibular retrusion". International Journal of Oral and Maxillofacial Surgery 34 (2005): 846-850.

46. Burstone J. "Integumental contour and extension patterns". American Journal of Orthodontics 29 (1959): 93-104.

47. Converse JM., et al. "Deformities of the jaws". In: Converse JM (edition) Reconstructive plastic surgery. 2nd ed. WB Saunders: Philadelphia (1977): 1386-1456.

48. Wolfe SA., et al. "The genioplasty and beyond: An end-game strategy for the multiply operated chin". Plastic and Reconstructive Surgery 117 (2006): 1435-1446.

49. Mittal G., et al. "The Art of Genioplasty: An Insight". International Journal of Oral Health and Medical Research 4.3 (2017): 86-94.

\section{Volume 5 Issue 10 October 2021} (C) All rights are reserved by Shashank Bhushan. 